\title{
TTR
}

Traduction, terminologie, rédaction

\section{Kyle Conway. Everyone Says No: Public Service Broadcasting and the Failure of Translation. Montreal, McGill-Queen's University Press, 2011, 217 p.}

\section{Chantal Gagnon}

Volume 25, numéro 2, 2e semestre 2012

URI : https://id.erudit.org/iderudit/1018811ar

DOI : https://doi.org/10.7202/1018811ar

Aller au sommaire du numéro

Éditeur(s)

Association canadienne de traductologie

ISSN

0835-8443 (imprimé)

1708-2188 (numérique)

Découvrir la revue

Citer ce compte rendu

Gagnon, C. (2012). Compte rendu de [Kyle Conway. Everyone Says No: Public Service Broadcasting and the Failure of Translation. Montreal, McGill-Queen's University Press, 2011, 217 p.] TTR, 25(2), 257-262.

https://doi.org/10.7202/1018811ar d'utilisation que vous pouvez consulter en ligne.

https://apropos.erudit.org/fr/usagers/politique-dutilisation/ 


\section{COMPTES RENDUS}

\section{Kyle Conway. Everyone Says No: Public Service Broadcasting and the Failure of Translation. Montreal, McGill-Queen's University Press, 2011, 217 p.}

Louvrage Everyone Says No, paru en 2011 aux éditions McGillQueen's University Press, porte sur le rôle de la traduction dans la télévision publique canadienne. Dans son étude, l'auteur vise tout particulièrement la couverture médiatique de Radio-Canada et de $\mathrm{CBC}$ lors de deux événements phares de l'histoire canadienne contemporaine, l'Accord du Lac Meech (1987) et l'Accord de Charlottetown (1992). Même si la contribution de Kyle Conway appartient d'abord au domaine de la communication (p. 8), son apport à la traductologie est lui aussi très important; pour des raisons évidentes, c'est de cette dernière dont nous traiterons dans le présent compte rendu. Louvrage recensé ici complète bien les travaux récents sur la traduction et les médias (Bielsa et Bassnett, 2009), en offrant une perspective institutionnelle canadienne.

Everyone Says No se divise en six chapitres, introduction et conclusion non comprises. En plus des sections traditionnelles comme les notes, la bibliographie et l'index, le livre présente aussi une annexe utile sur les dates importantes de l'histoire constitutionnelle canadienne.

$\mathrm{Au}$ tout début de l'ouvrage, le lecteur apprend que le titre Everyone Says No fait référence aux propos de Jacques Parizeau le 19 octobre 1992, avant le référendum sur l'Accord de Charlottetown : "Lundi prochain là, qu'est-ce qui arrive? Les autochtones disent non aux Canadiens anglais pis aux Canadiens français, les Canadiens français disent non aux Canadiens anglais [...], on se trouve tous - on se dit tous non » (p. 3, nous soulignons). L'introduction sert aussi à présenter le corpus, la méthodologie ainsi qu'à résumer le contenu du livre.

Le premier chapitre décrit le contexte sociohistorique du corpus, notamment en examinant le concept de "nation " et en étudiant le système médiatique canadien. S'il incombe à Radio-Canada et à $\mathrm{CBC}$ d'aider les deux solitudes canadiennes 
à s'apprivoiser l'une l'autre, il s'agit d'une mission entravée par des obstacles historiquement conditionnés. Malgré ces difficultés, l'auteur est d'avis que les deux diffuseurs ont les outils, du moins en théorie, pour amorcer la levée de ces obstacles. Une réussite à cet égard ferait sans doute de Radio-Canada et de CBC des modèles à suivre pour les autres diffuseurs publics (p. 21).

Le second chapitre porte entre autres sur un projet pilote du début des années 1990, où le réseau CBC diffusait Le Téléjournal de Radio-Canada avec des sous-titres anglais. Ce projet a été interrompu rapidement et l'expérience inverse, soit le sous-titrage en français du bulletin d'information The National, ne vit jamais le jour. L'échec de cette aventure est notamment attribué aux pressions exercées par les journalistes d'expression française, qui craignaient que le sous-titrage de l'émission The National en français ne nuise à leurs efforts d'obtenir une chaîne dédiée à l'information, similaire à celle qui existait au Canada anglais. De cette expérience, Conway tire la conclusion qu'au début des années 1990, le radiodiffuseur public a dû reconnaître que la traduction nétait pas apolitique, malgré son potentiel de rapprochement entre les communautés linguistiques du pays.

Le troisième chapitre étudie le contexte au sein duquel le journalisme est pratiqué à $\mathrm{CBC}$ et à Radio-Canada. On apprend ainsi que la traduction au National et au Téléjournal ne constitue pas un outil neutre : ainsi, toute parole prononcée en langue étrangère peut être transmise selon divers formats (par exemple, citation directe ou indirecte, sous-titrage ou traduction hors champ), ce qui a une incidence sur le sujet présenté. Conway rapporte que lors du traitement des déclarations en langue étrangère, les journalistes du National emploient surtout la citation indirecte alors que ceux du Téléjournal ont davantage recours à la citation directe (p. 79). Par ailleurs, les journalistes de la télévision publique font souvent appel à la traduction pour équilibrer les points de vue de leur exposé. Ainsi, dans un reportage donné, les locuteurs qui s'expriment dans l'autre langue officielle expriment également un point de vue qui diffère des autres intervenants. Avec ce genre de procédé, les auditeurs sont en mesure d'anticiper les propos de ces locuteurs et de ne pas leur prêter une attention soutenue. Dans ces circonstances, si l'utilisation de la traduction 
avait pour objectif d'amener les deux communautés linguistiques à mieux comprendre leurs perspectives respectives, il s'agit d'un échec de communication (p. 80).

Les chapitres 4, 5 et 6 portent sur la traduction de "société distincte " par « distinct society ». Conway avance que ces termes prenaient des significations très différentes au Téléjournal et au National. Le chapitre 4 suit l'évolution historique du terme "société distincte ", tandis que les chapitres 5 et 6 expliquent quel sens a été donné à " société distincte » et à " distinct society " lors des bulletins d'information sur les accords de Meech et de Charlottetown.

Louvrage de Conway porte non seulement sur un thème neuf, mais il présente une recherche fouillée, émaillée d'exemples et fort intéressante. Le sujet est bien découpé, l'analyse, intelligente et la rédaction, soignée. La bibliographie est riche, à jour et généralement pertinente. L'apport des travaux de Conway à la traductologie est par ailleurs indéniable, particulièrement dans le domaine de la traduction culturelle. Notre discipline peine encore à définir concrètement ce qu'est la traduction culturelle et sur ce point, Conway fait une excellente contribution. Pour ce chercheur, il y a traduction culturelle lorsque des journalistes expliquent à un groupe culturel comment un autre groupe culturel interprète un sujet ou un événement (p. 5). Conway montre bien, par exemple, que dans le contexte précis de la télévision publique canadienne, les rouages de production de la nouvelle ne sont pas les mêmes à Radio-Canada et à $\mathrm{CBC}$, ce qui a des répercussions importantes sur le traitement d'un même renseignement en français et en anglais. À ce chapitre, l'analyse de la traduction de "société distincte » est tout simplement fascinante. Certains quotidiens d'expression française avaient déjà affirmé que les termes « distinct society " et "société distincte " nétaient pas équivalents (Vastel, 1996, p. B7), mais il s'agissait de commentaires anecdotiques. Ici, Conway démontre comment et pourquoi ces termes renvoient à des réalités différentes. En fait, le sens de ces deux termes était notamment associé aux manœuvres des politiciens d'expression anglaise et française, afin de rendre leurs politiques acceptables dans les deux communautés linguistiques. 
Malgré les forces indéniables du livre, il y a certains aspects qui nous amènent à exprimer de toutes petites réserves. Par exemple, la traduction culturelle telle que définie ici se limite trop souvent à la situation des médias. Ce n'est que dans une note du chapitre 6 (p. 187, note 3) que l'auteur discute plus largement de la définition de traduction culturelle, en évoquant, à titre d'exemple, la façon dont cette définition est perçue dans les Cultural Studies. En situant dès le premier chapitre la traduction culturelle dans un contexte plus large, Conway aurait pu établir des liens avec des recherches similaires, donnant ainsi une plus grande portée à ses affirmations. La contribution d'Everyone Says No quant à la traduction culturelle n’en aurait été que plus riche.

Par ailleurs, au chapitre 3, les types de traduction répertoriés ne correspondent pas toujours aux définitions traditionnelles en traductologie (p. 70ff). Par exemple, rarement a-t-on décrit l'autotraduction comme un énoncé prononcé par un locuteur qui ne s'exprime pas dans sa langue maternelle; il s'agit davantage d'une déclaration en L2/L3. De même, on se demande quelle est la différence entre la traduction paraphrasée ("paraphrased translation») et la traduction résumée («summary translation »). Comme le souligne l'ouvrage The Metalanguage of Translation (Gambier et van Doorslaer, 2009), il faut tendre vers un métalangage consensuel afin de faciliter la compréhension des phénomènes de la traduction.

Il est également dommage qu'au chapitre 6, en abordant les discours politiques de Pierre Elliott Trudeau (p. 150), l'auteur fasse aussi peu référence à la question de la traduction. Ainsi, on mentionne le discours historique de Trudeau au restaurant la Maison du Egg Roll, sans préciser en quelle(s) langue(s) le discours s'est déroulé, ni si le résumé du National le soir même constituait une traduction. En outre, en note de bas de page (p. 187, note 1), Conway écrit que l'impopularité de Trudeau au Québec a sans doute eu des répercussions sur la réception de son texte publié en français dans l'Actualité. Nous ne mettons pas en doute cet énoncé, mais Conway aurait aussi pu faire ressortir que la réception des textes de Trudeau était également tributaire du contenu de la traduction. Dans la presse écrite, plusieurs journalistes ont déjà expliqué qu'il existait parfois des divergences 
entre les textes français et anglais de Trudeau. Des études en traductologie montrent en outre que les discours politiques canadiens renferment souvent un nombre appréciable d'écarts de traduction (Bánhegyi, 2008; Fraser, 2004), une caractéristique particulièrement présente chez Trudeau (Gagnon, 2006a, 2006b).

En terminant, Conway n'est pas le premier à se pencher sur les différences entre le discours journalistique du Québec et celui du Canada (nous pensons entre autres à Robinson, 1998), mais il est le premier à tenir compte du phénomène de la traduction et à le faire de façon aussi convaincante. Son ouvrage mérite franchement qu'on s'y attarde, ne serait-ce que pour mieux comprendre le rôle de la traduction chez les radiodiffuseurs de l'unifolié.

\section{Références}

BÁNHEGYI, Mátyás (2008). «A Translation Studies Oriented Integrative Approach to Canadian Political Discourse ». Across Languages and Cultures, 9, 1, pp. 77-107.

BIELSA, Esperança et Susan BASSNETT (2009). Translation in Global Nerws. Londres et New York, Routledge.

FRASER, Kelli (2004). Translating History: Prime Minister MacKenzie King's Speeches During the Conscription Crisis. Mémoire de maîtrise, École de traduction et d'interprétation, Université d'Ottawa. Inédit.

GAGNON, Chantal (2006a). "Ideologies in the History of Translation: a Case Study on Canadian Political Speeches ", in G. Bastin et P. Bandia, dir. Charting the Future of Translation Studies. Ottawa, University of Ottawa Press, pp. 201-223.

GAGNON, Chantal (2006b). "Language Plurality as Power Struggle, or:Translating Politics in Canada ». Target. International Journal of Translation Studies, 18, 1, pp. 69-90.

GAMBIER, Yves et Luc VAN DOORSLAER (2009). The Metalanguage of Translation. Amsterdam et Philadelphie, John Benjamins Publishing Company. 
ROBINSON, Gertrude Joch (1998). Constructing the Quebec Referendum : French and English Media Voices. Toronto, University of Toronto Press.

VASTEL, Michel (1996). " La patrie des francophones d'Amérique ». Le Soleil, 6 mai, p. B7.

Chantal Gagnon Université de Montréal

Yves Gambier et Luc van Doorslaer, dir. Handbook of Translation Studies. Volume 1. Amsterdam et Philadelphie, John Benjamins Publishing Company, 2010, 458 p.

Yves Gambier et Luc van Doorslaer, dir. Handbook of Translation Studies. Volume 2. Amsterdam et Philadelphie, John Benjamins Publishing Company, 2011, 197 p.

Yves Gambier et Luc van Doorslaer, dir. Handbook of Translation Studies Online. Amsterdam, John Benjamins Publishing Company, 2012 ( $2^{\mathrm{e}}$ éd.). Disponible à : http://www.benjamins. nl/online/hts/ [consulté le 3 août 2012].

La traductologie, affirment Yves Gambier et Luc van Doorslaer, a atteint un tel niveau de maturité institutionnelle qu'elle mérite, à titre de discipline, de faire l'objet de multiples ouvrages de référence : dictionnaires, encyclopédies, introductions et manuels. $\mathrm{Au}$ Routledge Encyclopedia of Translation Studies (Baker et Saldanha, 2011 [1998]), maintenant à sa deuxième édition, au Translation Studies en quatre volumes dirigé par Mona Baker (2009) et au Oxford Handbook of Translation Studies (Malmkjær et Windle, 2011), s'ajoute donc un nouvel ouvrage de référence, publié cette fois chez John Benjamins Publishing Company : Handbook of Translation Studies (Gambier et van Doorslaer, 2010; 2011).

Selon ses directeurs, le manuel offre deux avantages par rapport à ses compétiteurs : d'abord, il est publié à la fois sur papier (au rythme d'un volume par année) et en ligne (où les articles seront continuellement mis à jour) (www.benjamins.nl/ 